Bulletin of the Section of Logic

Volume 49/3 (2020), pp. 231-253

http://dx.doi.org/10.18778/0138-0680.2020.12

\title{
EMPIRICAL NEGATION, CO-NEGATION AND THE CONTRAPOSITION RULE I: SEMANTICAL INVESTIGATIONS
}

\begin{abstract}
We investigate the relationship between M. De's empirical negation in Kripke and Beth Semantics. It turns out empirical negation, as well as co-negation, corresponds to different logics under different semantics. We then establish the relationship between logics related to these negations under unified syntax and semantics based on R. Sylvan's $\mathbf{C C}_{\omega}$.

Keywords: Empirical negation, co-negation, Beth semantics, Kripke semantics, intuitionism.

\section{Introduction}

The philosophy of Intuitionism has long acknowledged that there is more to negation than the customary, reduction to absurdity. Brouwer [1] has already introduced the notion of apartness as a positive version of inequality, such that from two apart objects (e.g. points, sequences) one can learn not only they are unequal, but also how much or where they are different. (cf. $[19$, pp.319-320]). He also introduced the notion of weak counterexample, in which a statement is reduced to a constructively unacceptable principle, to conclude we cannot expect to prove the statement [17].
\end{abstract}

Presented by: Andrzej Indrzejczak

Received: April 18, 2020

Published online: August 15, 2020

(C) Copyright for this edition by Uniwersytet Łódzki, Łódź 2020 
Another type of negation was discussed in the dialogue of Heyting [8, pp. 17-19]. In it mathematical negation characterised by reduction to absurdity is distinguished from factual negation, which concerns the present state of our knowledge. In the dialogue it is emphasised that only the former type of negation has a part in mathematics, on the ground that the latter does not have the form of a mathematical assertion, i.e. assertion of a mental construction. Nevertheless it remains the case that factual negation has a place in his theoretical framework.

One formalisation of logic with this "negation at the present stage of knowledge" was given by De [3] and axiomatised by De and Omori [4], under the name of empirical negation. The central idea of $\mathbf{I P C} \sim$ is semantic: the Kripke semantics of $\mathbf{I P C}^{\sim}$ is taken to be rooted, with the root being understood as representing the present moment. Then the empirical negation $\sim A$ is defined to be forced at a world, if $A$ is not forced at the root.

Yet another type of negation in the intuitionistic framework is conegation introduced by Rauszer $[12,13]$. Seen from Kripke semantics, a co-negation $\sim A$ is forced at a world, if there is a preceding world in which $A$ is not forced. This is dual to the forcing of intuitionistic negation $\neg A$, which requires $A$ not being forced at all succeeding nodes. Co-negation was originally defined in terms of co-implication, but the co-negative fragment was extracted by Priest [11], to define a logic named daC.

In both empirical and co- negation, the semantic formulation arguably gives a more fundamental motivation than the syntactic formulation. In particular, in case of empirical negation, it is of essential importance that a Kripke frame can be understood as giving the progression of growth of knowledge. It may be noted, however, that Kripke semantics is not the only semantics to give this kind of picture. Beth semantics is another semantics whose frames represent the growth of knowledge. It then appears a natural question to ask, whether the same forcing condition of empirical/co- negation gives rise to the same logic. That is to say, whether $\mathbf{I P C}^{\sim}$ and $\mathbf{d a C}$ will be sound and complete with respect to Beth semantics. Indeed, for co-implication, a similar question was asked by Restall [14]. There it was found out that one needs to alter the forcing condition to get a complete semantics.

In this paper, we shall observe that another logic called $\mathbf{T} \mathbf{C C}_{\omega}$, introduced by Gordienko [7], becomes sound and complete with Beth models with the forcing conditions of empirical and co- negation (which turn out 
to coincide). This is of significant interest for those who advocate empirical or co- negation from a semantic motivation, as it will provide a choice in the logic to which they should adhere.

This is followed by another observation about the axiomatisation of $\mathbf{I P C}^{\sim}$ and $\mathbf{d a C}$, which employ the disjunctive syllogism rule $[\mathrm{RP}]$. In contrast, the axiomatisation of of $\mathbf{T} \mathbf{C C} \omega$ and a related system $\mathbf{C C}_{\omega}$ of Sylvan [15], which is a subsystem of the other three, use the contraposition rule $[\mathrm{RC}]$. We shall observe that this difference in rules can be eliminated, by replacing $[\mathrm{RP}]$ with $[\mathrm{RC}]$ and an additional axiom. This will give a completeness proof of $\mathbf{d a C}$ with respect to the semantics of $\mathbf{C C}_{\omega}$, and thus the semantics of Došen [5]. It will also provide a more unified viewpoint of the logics related to $\mathbf{C C}_{\omega}$ as defined by extra axioms with no change in rules.

We shall continue our investigation proof-theoretically in a sequel. In the second paper, using the obtained frame properties we shall formulate labelled sequent calculi for the logics considered so far $\left(\mathbf{C C}_{\omega}, \mathbf{d a C}, \mathbf{T} \mathbf{C C}_{\omega}\right.$ and $\mathbf{I P C} \sim$ ). We shall prove the admissibility of structural rules including cut, and then show the correspondence with Hilbert-style calculi.

\section{Preliminaries}

We shall employ the following notations (taken from [17]) for sequences and related notions.

- $\alpha, \beta, \ldots$ infinite sequences of the form $\left\langle b_{1}, b_{2}, \ldots\right\rangle$ of natural numbers.

- \langle\rangle : the empty sequence.

- $b, b^{\prime}, \ldots$ f finite sequences of the form $\left\langle b_{1}, \ldots, b_{n}\right\rangle$ of natural numbers.

- $b * b^{\prime}: b$ concatenated with $b^{\prime}$.

- $\operatorname{lh}(b)$ : the length of $b$.

- $b \preceq b^{\prime}: b * b^{\prime \prime}=b^{\prime}$ for some $b^{\prime \prime}$.

- $b \prec b^{\prime}: b \preceq b^{\prime}$ and $b \neq b^{\prime}$.

- $\bar{\alpha} n$ : $\alpha$ 's initial segment up to the $n$th element.

- $\alpha \in b: b$ is $\alpha$ 's initial segment.

We define a tree to be a set $T$ of finite sequences of natural number such that \langle\rangle$\in T, b \in T \vee b \notin T$ and $b \in T \wedge b^{\prime} \prec b \rightarrow b^{\prime} \in T$. We call each finite 
sequence in $T$ a node and \langle\rangle the root. A successor of a node $b$ is a node of the form $b *\langle x\rangle$. By leaves of $T$, we mean the nodes of $T$ which do not have a successor, i.e. nodes $b$ such that $\neg \exists x(b *\langle x\rangle) \in T$. A spread then is a tree whose nodes always have a successor, i.e. $\forall b \in T \exists x(b *\langle x\rangle \in T)$.

A clarification: whilst $\langle b, b, \ldots\rangle$ denotes an infinite sequence consisting just of $b \mathrm{~s},\langle b, \ldots, b\rangle$ denotes a finite sequence consisting just of $b \mathrm{~s}$.

\section{Empirical negation in Kripke Semantics}

Let us use the following notations for metavariables.

- $p, q, r, \ldots$ for propositional variables.

- $A, B, C, \ldots$ for formulae.

In this paper, we shall consider the following propositional language

$$
\mathcal{L}::=p|(A \wedge B)|(A \vee B)|(A \rightarrow B)| \sim A .
$$

Parentheses will be omitted if there is no fear of ambiguity. We shall use the convention $A \leftrightarrow B:=(A \rightarrow B) \wedge(B \rightarrow A)$.

To begin with, we look at the Kripke semantics for the intuitionistic logic with empirical negation $\mathbf{I P C}^{\sim}$ given in [4]. Recall that a reflexive, anti-symmetric and transitive ordering is called a partial order.

Definition 3.1 (Kripke model for IPC ${ }^{\sim}$ ). A Kripke Frame $\mathcal{F}_{\mathcal{K}}$ for $\mathbf{I P C}^{\sim}$ is a partially ordered set $(W, \leq)$ with a root $r \in W$ such that $r \leq w$ for all $w \in W$. We shall call each $w \in W$ a world. A Kripke model $\mathcal{M}_{\tilde{\mathcal{K}}}$ for IPC $^{\sim}$ is a pair $\left(\mathcal{F}_{\mathcal{K}}, \mathcal{V}\right)$, where $\mathcal{V}$ is a mapping that assigns a set of worlds $\mathcal{V}(p) \subseteq W$ to each propositional variable $p$. We assume $\mathcal{V}$ to be monotone, viz. $w \in \mathcal{V}(p)$ and $w^{\prime} \geq w$ implies $w^{\prime} \in \mathcal{V}(p)$. To denote a model, we shall use both $\mathcal{M}_{\tilde{\mathcal{K}}}$ and $\left(\mathcal{F}_{\mathcal{K}}, \mathcal{V}\right)$ interchangeably. Similar remarks apply to different notions of model in the later sections.

Given $\mathcal{M}_{\mathcal{K}}$, the forcing (or valuation) of a formula in a world, denoted $\mathcal{M}_{\mathcal{K}}^{\tilde{\mathcal{K}}}, w \Vdash_{\mathcal{K}} A$, is inductively defined as follows. 


$$
\begin{aligned}
& \mathcal{M}_{\mathcal{K}}^{\tilde{\mathcal{K}}}, w \Vdash_{\mathcal{K}} p \quad \Longleftrightarrow w \in \mathcal{V}(p) . \\
& \mathcal{M}_{\mathcal{K}}^{\tilde{\mathcal{K}}}, w \Vdash_{\mathcal{K}} A \wedge B \Longleftrightarrow \mathcal{M}_{\mathcal{K}}^{\tilde{\mathcal{K}}}, w \Vdash_{\mathcal{K}} A \text { and } \mathcal{M}_{\mathcal{K}}^{\tilde{\mathcal{K}}}, w \Vdash_{\mathcal{K}} B \text {. } \\
& \mathcal{M}_{\mathcal{K}}^{\tilde{\mathcal{K}}}, w \Vdash_{\mathcal{K}} A \vee B \Longleftrightarrow \mathcal{M}_{\mathcal{K}}^{\tilde{\mathcal{K}}}, w \Vdash_{\mathcal{K}} A \text { or } \mathcal{M}_{\mathcal{K}}^{\tilde{\mathcal{K}}}, w \Vdash_{\mathcal{K}} B \text {. } \\
& \mathcal{M}_{\mathcal{K}}, w \Vdash_{\mathcal{K}} A \rightarrow B \Longleftrightarrow \text { for all } w^{\prime} \geq w \text {, if } \mathcal{M}_{\mathcal{K}}, w^{\prime} \Vdash_{\mathcal{K}} A \text {, } \\
& \text { then } \mathcal{M}_{\mathcal{K}}, w^{\prime} \Vdash_{\mathcal{K}} B \text {. } \\
& \mathcal{M}_{\mathcal{K}}^{\tilde{\mathcal{K}}}, w \Vdash_{\mathcal{K}} \sim A \quad \Longleftrightarrow \mathcal{M}_{\mathcal{K}}^{\tilde{\mathcal{K}}}, r \nVdash_{\mathcal{K}} A \text {. }
\end{aligned}
$$

We shall occasionally avoid denoting models explicitly when it is apparent from the context. If $\mathcal{M}_{\mathcal{K}}, w \Vdash_{\mathcal{K}} A$ for all $w \in W$, we write $\mathcal{M}_{\mathcal{\mathcal { K }}} \vDash_{\mathcal{K}} A$ and say $A$ is valid in $\mathcal{M}_{\mathcal{K}}$. For a set of formulae $\Gamma$, if $\mathcal{M}_{\mathcal{K}} \vDash_{\mathcal{K}} C$ for all $C \in \Gamma$ implies $\mathcal{M}_{\mathcal{K}} \vDash_{\mathcal{K}} A$, then we write $\Gamma \vDash_{\mathcal{K}} A$ and say $A$ is a consequence of $\Gamma$. If $\Gamma$ is empty, we simply write $\vDash_{\mathcal{K}} A$ and say $A$ is valid (in $\mathbf{I P C}^{\sim}$ ).

A Hilbert-style proof system for $\mathbf{I P C}^{\sim}$ is established in [4], which we identify here with the logic itself for convenience, and denote it simply as $\mathbf{I P C}^{\sim}$. We shall apply the same convention to other logics in later sections.

DEFINITION $3.2\left(\right.$ IPC $\left.^{\sim}\right)$.

The logic $\mathbf{I P C}^{\sim}$ is defined by the following axiom schemata and rules.

$\underline{\text { Axioms }}$
$[\mathrm{Ax} 1]$

$$
A \rightarrow(B \rightarrow A)
$$
[Ax2]
$(A \rightarrow(B \rightarrow C)) \rightarrow((A \rightarrow B) \rightarrow(A \rightarrow C))$
$[\mathrm{Ax} 3]$

$$
(A \wedge B) \rightarrow A
$$
$[\mathrm{Ax} 4]$$$
(A \wedge B) \rightarrow B
$$
$[\mathrm{Ax} 5]$
$[\mathrm{Ax} 6]$
$(C \rightarrow A) \rightarrow((C \rightarrow B) \rightarrow(C \rightarrow(A \wedge B)))$
[Ax7]

$$
A \rightarrow(A \vee B)
$$
$[\mathrm{Ax} 8]$

$$
B \rightarrow(A \vee B)
$$
[Ax9]

$$
(A \rightarrow C) \rightarrow((B \rightarrow C) \rightarrow((A \vee B) \rightarrow C))
$$
[Ax10]

$$
A \vee \sim A
$$$$
\sim A \rightarrow(\sim \sim A \rightarrow B)
$$

\section{$\underline{\text { Rules }}$}

$$
[\mathrm{MP}] \frac{A \quad A \rightarrow B}{B} \quad[\mathrm{RP}] \frac{A \vee B}{\sim A \rightarrow B}
$$


We followed [4] in the labelling of the axioms and the rules. A proof (or deduction/derivation) of $A$ from a (possibly infinite) set of formulae $\Gamma$ (which we denote by $\Gamma \vdash \sim A$ ) in $\mathbf{I P C}^{\sim}$ is a finite tree with the number of branching at each node less than or equal two, and whose nodes are labelled by formulae of $\mathcal{L}$ such that

- The formulae in the leaves are either instances of axioms, or from a specified finite subset $\Gamma^{\prime}$ of $\Gamma$.

- Each formula in non-leaf nodes is obtained from the formulae in the successor nodes by an application of a rule.

- The root of the tree is $A$.

Then it has been shown by De and Omori that IPC ${ }^{\sim}$ is sound and complete with the Kripke semantics.

TheOREM 3.3 (Kripke completeness of IPC $^{\sim}$ ). $\Gamma \vdash \sim A \Longleftrightarrow \Gamma \vDash_{\mathcal{K}} A$.

Proof: Cf. [4].

\section{Empirical negation in Beth Semantics}

\subsection{Beth semantics and IPC $\sim$}

Let us turn our attention to Beth models in this section. Our formalisation will be based on that of $[16,18]$. If we apply to the forcing of $\sim$ the same criterion as to the Kripke semantics above, then we obtain the following semantics.

Definition 4.1 (Beth model). A Beth frame $\mathcal{F}_{\mathcal{B}}$ is a pair $(W, \preceq)$ that defines a spread. Then A Beth model $\mathcal{M}_{\mathcal{B}}$ is a pair $\left(\mathcal{F}_{\mathcal{B}}, \mathcal{V}\right)$, where $\mathcal{V}$ is an assignment of propositional variables to the nodes such that:

$$
b \in \mathcal{V}(p) \Leftrightarrow \forall \alpha \in b \exists m(\bar{\alpha} m \in \mathcal{V}(p)) . \text { [covering] }
$$

(The left-to-right direction is trivial, and it is straightforward to see that a covering assignment is monotone.)

The forcing relation $\vdash_{\mathcal{B}} A$ for a Beth model is defined by the following clauses. 


$$
\begin{aligned}
\mathcal{M}_{\mathcal{B}}, b \Vdash_{\mathcal{B}} p & \Longleftrightarrow b \in \mathcal{V}(p) . \\
\mathcal{M}_{\mathcal{B}}, b \Vdash_{\mathcal{B}} A \wedge B & \Longleftrightarrow \mathcal{M}_{\mathcal{B}}, b \Vdash_{\mathcal{B}} A \text { and } \mathcal{M}_{\mathcal{B}}, b \Vdash_{\mathcal{B}} B . \\
\mathcal{M}_{\mathcal{B}}, b \vdash_{\mathcal{B}} A \vee B & \Longleftrightarrow \forall \alpha \in b \exists n\left(\mathcal{M}_{\mathcal{B}}, \bar{\alpha} n \Vdash_{\mathcal{B}} A \text { or } \mathcal{M}_{\mathcal{B}}, \bar{\alpha} n \Vdash_{\mathcal{B}} B\right) . \\
\mathcal{M}_{\mathcal{B}}, b \Vdash_{\mathcal{B}} A \rightarrow B & \Longleftrightarrow \text { for all } b^{\prime} \succeq b, \text { if } \mathcal{M}_{\mathcal{B}}, b^{\prime} \Vdash_{\mathcal{B}} A, \text { then } \mathcal{M}_{\mathcal{B}}, b^{\prime} \Vdash_{\mathcal{B}} B . \\
\mathcal{M}_{\mathcal{B}}, b \Vdash_{\mathcal{B}} \sim A & \Longleftrightarrow \mathcal{M}_{\mathcal{B}},\langle\rangle \nVdash_{\mathcal{B}} A .
\end{aligned}
$$

Proposition 4.2.

(i) $b \Vdash_{\mathcal{B}} A$ if and only if $\forall \alpha \in b \exists n\left(\bar{\alpha} n \Vdash_{\mathcal{B}} A\right)$. (covering property)

(ii) $b^{\prime} \succeq b$ and $b \Vdash_{\mathcal{B}} A$ implies $b^{\prime} \Vdash_{\mathcal{B}} A$. (monotonicity)

Proof: We prove (i) by induction on the complexity of formulae. If $b \Vdash_{\mathcal{B}}$ $A$, then trivially $\forall \alpha \in b \exists n\left(\bar{\alpha} n \Vdash_{\mathcal{B}} A\right)$. For the converse direction, we show by induction on the complexity of $A$. Because (i) holds in Beth models for intuitionistic logic, it suffices to check the case where $A \equiv \sim B$. If $\forall \alpha \in b \exists n\left(\bar{\alpha} n \Vdash_{\mathcal{B}} \sim B\right)$, then by definition $\forall \alpha \in b \exists n\left(\langle\rangle \nVdash_{\mathcal{B}} B\right)$; i.e. \langle\rangle$\nVdash_{\mathcal{B}} B$. Thus by definition again, $b \Vdash_{\mathcal{B}} \sim B$.

(ii) is an immediate consequence of (i).

How does this semantics relate to IPC ${ }^{\sim}$ ? In considering this question, we first look at how to embed Kripke models into Beth models, in accordance with the method outlined in [18].

Given a Kripke model $\mathcal{M}_{\mathcal{K}}=\left(W_{K}, \leq, \mathcal{V}_{K}\right)$ for $\mathbf{I P C}^{\sim}$, we construct a corresponding Beth model $\mathcal{M}_{\mathcal{B}}=\left(W_{B}, \preceq, \mathcal{V}_{B}\right)$ with the following stipulations.

- $W_{B}$ is the set of finite nondecreasing sequences of worlds (i.e. each $w$ in a sequence is followed by $w^{\prime}$ s.t. $w \leq w^{\prime}$ ) from the root $r$ in $\left(W_{K}, \leq\right)$ with length $>0$.

- $\preceq$ is defined accordingly.

- $\left\langle w_{0}, \ldots, w_{n}\right\rangle \in \mathcal{V}_{B}(p)$ if and only if $w_{n} \in \mathcal{V}_{K}(p)$.

The resulting $W_{B}$ is a spread, because the reflexivity of $\leq$ assures that $\left\langle w_{0}, \ldots, w_{n}\right\rangle \in W_{B}$ implies $\left\langle w_{0}, \ldots, w_{n}, w_{n}\right\rangle \in W_{B}$. Note that $w_{0}$ is always the root $r$ in $\mathcal{M}_{\mathcal{K}}$, and $\left\langle w_{0}\right\rangle$ is the root of $\mathcal{M}_{\mathcal{B}}$. The latter slightly differs from our definition of Beth model: we can fit the model to the definition if we reinterpret the sequences as mere labels for the tree, and the actual 
tree is constructed in such a way that $\left\langle w_{0}\right\rangle$ is the label for the node \langle\rangle , $\left\langle w_{0}, w_{1}, \ldots, w_{n}\right\rangle$ is the label for the node $\left\langle w_{1}, \ldots, w_{n}\right\rangle$. We can also adopt a different embedding, which we shall see later.

For any Kripke model, because we can concatenate the same element indefinitely many times, we can also consider infinite nondecreasing sequences of worlds. This fact will be used in the next lemma.

LEMma 4.3 (embeddability of Kripke models for $\mathbf{I P C}^{\sim}$ ).

(i) $\mathcal{M}_{\mathcal{B}}$ is indeed a Beth model.

(ii) $\mathcal{M}_{\mathcal{K}}^{\tilde{K}} \vDash_{\mathcal{K}} A$ if and only $\mathcal{M}_{\mathcal{B}} \vDash_{\mathcal{B}} A$.

Proof: For (i), we need to check that $\mathcal{V}_{B}$ is a covering assignment. If $\forall \alpha \in$ $\left\langle w_{0}, \ldots, w_{n}\right\rangle \exists m\left(\bar{\alpha} m \in \mathcal{V}_{B}(p)\right)$, then in particular, $\alpha_{0}:=\left\langle w_{0}, \ldots, w_{n}\right\rangle *$ $\left\langle w_{n}, w_{n}, \ldots\right\rangle \in\left\langle w_{0}, \ldots, w_{n}\right\rangle$. So there is an $m$ such that $\overline{\alpha_{0}} m \in \mathcal{V}_{B}(p)$. If $m \leq n+1=l h\left(\left\langle w_{0}, \ldots, w_{n}\right\rangle\right)$, then by the monotonicity of $\mathcal{V}_{B}$ (which follows from that of $\mathcal{V}_{K}$, and the fact that $\mathcal{V}_{B}$ only looks at the last element of a sequence) we have $\left\langle w_{0}, \ldots, w_{n}\right\rangle \in \mathcal{V}_{B}(p)$. Otherwise, by definition of $\mathcal{V}_{B}, w_{n} \in \mathcal{V}_{K}(p)$; hence $\left\langle w_{0}, \ldots, w_{n}\right\rangle \in \mathcal{V}_{B}(p)$.

For (ii), it suffices to show $w_{n} \Vdash_{\mathcal{K}} A \Leftrightarrow\left\langle w_{0}, \ldots, w_{n}\right\rangle \Vdash_{\mathcal{B}} A$. We prove this by induction on the complexity of formulae. Given the result for intuitionistic logic, we only need to check for $A \equiv \sim B$. In this case, $w_{n} \Vdash_{\mathcal{K}} \sim B \Leftrightarrow w_{0} \nVdash_{\mathcal{K}} B \Leftrightarrow\left\langle w_{0}\right\rangle \nVdash_{\mathcal{B}} B \Leftrightarrow\left\langle w_{0}, \ldots, w_{n}\right\rangle \Vdash_{\mathcal{B}} \sim B$.

Let $\mathrm{Q}$ be the class of Beth models obtained by the above embedding. We shall denote Beth validity with respect to $Q$ as $\vDash_{Q}$.

TheOrem 4.4 (Beth completeness of $\mathbf{I P C}^{\sim}$ with respect to $\mathrm{Q}$ ). $\Gamma \vdash \sim A$ if and only if $\Gamma \vDash_{\mathrm{Q}} A$.

Proof: Because of Theorem 3.3, $\Gamma \vdash \sim A$ if and only if $\Gamma \vDash_{\mathcal{K}} A$. Also by the preceding lemma, $\Gamma \vDash_{\mathcal{K}} A$ if and only if $\Gamma \vDash_{\mathrm{Q}} A$.

\subsection{Beth Semantics and $\mathrm{TCC}_{\omega}$}

The above theorem shows that $\mathbf{I P C}{ }^{\sim}$ is sound and complete with respect to a certain class of Beth models. The question remains, however, of whether it is sound and complete with respect to all Beth models. A problem lies in the soundness direction, of the validity of $[\mathrm{RP}]$. In a Beth model, it is possible that a disjunction is forced at a world whilst neither of the disjuncts is. 
This is contrastable with an admissible [4] rule $[\mathrm{RC}] \frac{A \rightarrow B}{\sim B \rightarrow \sim A}$ of IPC $^{\sim}$. Given any Beth model and assuming $A \rightarrow B$ is valid, if $\sim B$ is forced at a node $b^{\prime} \succeq b$ given an arbitrary $b$, then \langle\rangle does not force $B$, so \langle\rangle cannot force $A$ either; thus we can conclude $b^{\prime}$ forces $\sim A$ and so $b$ forces $\sim B \rightarrow \sim A$, i.e. $\sim B \rightarrow \sim A$ is valid.

This admissibility of $[\mathrm{RC}]$ in Beth models motivates us to consider a variant of $\mathbf{I P C}^{\sim}$ in which $[\mathrm{RP}]$ is replaced with $[\mathrm{RC}]$. As already mentioned in [4], such a logic is known under the name $\mathbf{T C C} \mathbf{C}_{\omega}$, formulated by Gordienko in [7].

Definition $4.5\left(\mathbf{T C C}_{\omega}\right)$. $\mathbf{T C C}_{\omega}$ is defined by axioms [Ax1] to [Ax10], and rules $[\mathrm{MP}]$ and $[\mathrm{RC}] \frac{A \rightarrow B}{\sim B \rightarrow \sim A}$.

We shall denote the provability in $\mathbf{T C C}_{\omega}$ by $\vdash_{t}$. We shall prove the soundness and completeness of $\mathbf{T} \mathbf{C C} \omega$ with respect to all Beth models. Again we want to embed Kripke models into Beth models; but as we see below, the Kripke models for $\mathbf{T} \mathbf{C C}_{\omega}$ are not necessarily rooted. So we shall embed models in a slightly different way.

Definition 4.6 (Kripke model for $\mathbf{T C C} \omega$ ). A Kripke Frame $\mathcal{F}_{\mathcal{K}}^{t}=(W, \leq$ ) for $\mathbf{T} \mathbf{C C}_{\omega}$ is a non-empty partially ordered set. A Kripke model $\mathcal{M}_{\mathcal{K}}^{t}$ for $\mathbf{T C C}_{\omega}$ is a pair $\left(\mathcal{F}_{\mathcal{K}}^{t}, \mathcal{V}\right)$, where $\mathcal{V}$ is a monotone mapping that assigns a set of worlds $\mathcal{V}(p) \subseteq W$ for each propositional variable $p$.

Given $\mathcal{M}_{\mathcal{K}}^{t}$, The forcing of a formula in a world, denoted $\mathcal{M}_{\mathcal{K}}^{t}, w \Vdash_{\mathcal{K} t} A$, is inductively defined as follows.

$$
\begin{aligned}
& \mathcal{M}_{\mathcal{K}}^{t}, w \Vdash_{\mathcal{K} t} p \Longleftrightarrow w \in \mathcal{V}(p) . \\
& \mathcal{M}_{\mathcal{K}}^{t}, w \Vdash_{\mathcal{K} t} A \wedge B \Longleftrightarrow \mathcal{M}_{\mathcal{K}}^{t}, w \Vdash_{\mathcal{K} t} A \text { and } \mathcal{M}_{\mathcal{K}}^{t}, w \Vdash_{\mathcal{K} t} B . \\
& \mathcal{M}_{\mathcal{K}}^{t}, w \Vdash_{\mathcal{K} t} A \vee B \Longleftrightarrow \mathcal{M}_{\mathcal{K}}^{t}, w \Vdash_{\mathcal{K} t} A \text { or } \mathcal{M}_{\mathcal{K}}^{t}, w \Vdash_{\mathcal{K} t} B . \\
& \mathcal{M}_{\mathcal{K}}^{t}, w \Vdash_{\mathcal{K}_{t}} A \rightarrow B \Longleftrightarrow \text { for all } w^{\prime} \geq w, \text { if } \mathcal{M}_{\mathcal{K}}^{t}, w^{\prime} \Vdash_{\mathcal{K}_{t}} A, \\
& \text { then } \mathcal{M}_{\mathcal{K}}^{t}, w^{\prime} \Vdash_{\mathcal{K} t} B . \\
& \mathcal{M}_{\mathcal{K}}^{t}, w \Vdash_{\mathcal{K} t} \sim A \quad \Longleftrightarrow \mathcal{M}_{\mathcal{K}}^{t}, w^{\prime} \nVdash_{\mathcal{K} t} A \text { for some } w^{\prime} .
\end{aligned}
$$

Theorem 4.7 (Kripke completeness for $\mathbf{T C C}_{\omega}$ ). $\vdash_{t} A$ if and only if $\vDash_{\mathcal{K} t} A$. Proof: Cf. [7]

Given a Kripke model $\mathcal{M}_{\mathcal{K}}^{t}=\left(W_{K}, \leq, \mathcal{V}_{K}\right)$ for $\mathbf{T} \mathbf{C C}_{\omega}$, we construct a corresponding Beth model $\mathcal{M}_{\mathcal{B}}=\left(W_{B}, \preceq, \mathcal{V}_{B}\right)$ with the following stipulation. 
- $W_{B}$ is the set of finite nondecreasing sequences in $\left(W_{K}, \leq\right)$ of length $\geq 0$.

- $\preceq$ is defined accordingly.

- Define an auxiliary valuation $\overline{\mathcal{V}}_{B}(p)$ s.t. $\left\langle w_{0}, \ldots w_{n}\right\rangle \in \overline{\mathcal{V}}_{B}(p)$ if and only if $w_{n} \in \mathcal{V}_{K}(p)$.

- Then $\mathcal{V}_{B}(p)=\overline{\mathcal{V}}_{B}(p) \cup\{\langle\rangle\}$ if $\mathcal{V}_{K}(p)=W_{K}$; otherwise $\mathcal{V}_{B}(p)=\overline{\mathcal{V}}_{B}(p)$.

LEMma 4.8 (embeddability of Kripke models for $\mathbf{T} \mathbf{C C}_{\omega}$ ).

(i) $\mathcal{M}_{\mathcal{B}}$ is indeed a Beth model.

(ii) $\mathcal{M}_{\mathcal{K}}^{t} \vDash_{\mathcal{K} t} A$ if and only $\mathcal{M}_{\mathcal{B}} \vDash_{\mathcal{B}} A$.

PROOF: In the following, we shall occasionally write $\left\langle b_{0}, \ldots, b_{-1}\right\rangle$ to mean \langle\rangle . (This is purely a conventional notation to simplify the exposition, and should not be confused with the notation in the definition of $\overline{\mathcal{V}}_{B}(p)$, in which $n$ cannot be -1 .)

(i) We need to show that the assignment is covering. Suppose $\left\langle b_{0}, \ldots, b_{n}\right\rangle$ $\in \mathcal{V}_{B}(p)$. If $n=-1$, then \langle\rangle$\in \mathcal{V}_{B}(p)$. So by definition of $\mathcal{V}_{B}$, $w \in \mathcal{V}_{K}(p)$ for all $w \in W_{K}$. Hence for each $\alpha=\langle w, \ldots\rangle \in\langle\rangle,\langle w\rangle \in$ $\mathcal{V}_{B}(p)$; so $\exists m\left(\bar{\alpha} m \in \mathcal{V}_{B}(p)\right)$. If $n>-1$, then $\left\langle b_{0}, \ldots, b_{n}\right\rangle \in \mathcal{V}_{B}(p)$ immediately implies $\forall \alpha \in\left\langle b_{0}, \ldots, b_{n}\right\rangle \exists m\left(\bar{\alpha} m \in \mathcal{V}_{B}(p)\right)$.

Conversely, suppose $\forall \alpha \in\left\langle b_{0}, \ldots, b_{n}\right\rangle \exists m\left(\bar{\alpha} m \in \mathcal{V}_{B}(p)\right)$. If $n=-1$, then for any $w \in W_{K},\langle w, w, \ldots\rangle \in\langle\rangle$. By our supposition, either \langle\rangle$\in \mathcal{V}_{B}(p)$ or $\langle w, w, \ldots, w\rangle \in \mathcal{V}_{B}(p)$. In both cases, $w \in \mathcal{V}_{K}(p)$. Hence $W_{K}=\mathcal{V}_{K}(p)$. Thus \langle\rangle$\in \mathcal{V}_{B}(p)$, as required. If $n>-1$, then $\left\langle b_{0}, \ldots, b_{n}, b_{n}, \ldots\right\rangle \in\left\langle b_{0}, \ldots, b_{n}\right\rangle$. So either \langle\rangle$\in \mathcal{V}_{B}(p),\left\langle b_{0}, \ldots, b_{i}\right\rangle \in$ $\mathcal{V}_{B}(p)$ for $i<n$, or $\left\langle b_{0}, \ldots, b_{n}, b_{n}, \ldots, b_{n}\right\rangle \in \mathcal{V}_{B}(p)$. In the first case, $b_{n} \in \mathcal{V}_{K}(p)$. In the second case, $b_{i} \in \mathcal{V}_{K}(p)$, so by the monotonicity of $\mathcal{V}_{K}, b_{n} \in \mathcal{V}_{K}$. In the last case, $b_{n} \in \mathcal{V}_{K}(p)$. So in any case, $\left\langle b_{0}, \ldots, b_{n}\right\rangle \in \mathcal{V}_{B}(p)$.

(ii) It suffices to show:

(a) \langle\rangle$\Vdash_{\mathcal{B}} A$ if and only if $\mathcal{M}_{\mathcal{K}}^{t} \vDash_{\mathcal{K} t} A$.

(b) $\left\langle b_{0}, \ldots, b_{n}\right\rangle \Vdash_{\mathcal{B}} A$ if and only if $b_{n} \Vdash_{\mathcal{K}_{t}} A$. (where $n>-1$ )

We prove these by simultaneous induction on the complexity of $A$. 
If $A \equiv p$, then 1 . and 2 . follow by definition.

If $A \equiv A_{1} \wedge A_{2}$, then for $1 .\langle\rangle \Vdash_{\mathcal{B}} A_{1} \wedge A_{2}$ if and only if \langle\rangle$\Vdash_{\mathcal{B}} A_{1}$ and \langle\rangle$\Vdash_{\mathcal{B}} A_{2}$ By I.H. this is equivalent to $\mathcal{M}_{\mathcal{K}}^{t} \vDash_{\mathcal{K} t} A_{1}$ and $\mathcal{M}_{\mathcal{K}}^{t} \vDash_{\mathcal{K} t} A_{2}$, which in turn is equivalent to $\mathcal{M}_{\mathcal{K}}^{t} \vDash_{\mathcal{K} t} A_{1} \wedge A_{2}$. For $2 .,\left\langle b_{0}, \ldots, b_{n}\right\rangle \Vdash_{\mathcal{B}}$ $A_{1} \wedge A_{2}$ if and only if $\left\langle b_{0}, \ldots, b_{n}\right\rangle \Vdash_{\mathcal{B}} A_{1}$ and $\left\langle b_{0}, \ldots, b_{n}\right\rangle \Vdash_{\mathcal{B}} A_{2}$. By I.H. this is equivalent to $b_{n} \Vdash_{\mathcal{K} t} A_{1}$ and $b_{n} \Vdash_{\mathcal{K} t} A_{2}$, which in turn is equivalent to $b_{n} \Vdash_{\mathcal{K} t} A_{1} \wedge A_{2}$.

If $A \equiv A_{1} \vee A_{2}$, then for 1., \langle\rangle$\Vdash_{\mathcal{B}} A_{1} \vee A_{2}$ if and only if $\forall \alpha \in$ \langle\rangle$\exists m\left(\bar{\alpha} m \Vdash_{\mathcal{B}} A_{1}\right.$ or $\left.\bar{\alpha} m \Vdash_{\mathcal{B}} A_{2}\right)$. For each $w \in W_{K},\langle w, w, \ldots\rangle \in\langle\rangle$, so either \langle\rangle$\Vdash_{\mathcal{B}} A_{1},\langle\rangle \Vdash_{\mathcal{B}} A_{2},\langle w, \ldots, w\rangle \Vdash_{\mathcal{B}} A_{1}$ or $\langle w, \ldots, w\rangle \Vdash_{\mathcal{B}} A_{2}$. If one of the former two cases holds, then by I.H. $\mathcal{M}_{\mathcal{K}}^{t} \vDash_{\mathcal{K} t} A_{i}$, for one of $i \in\{1,2\}$; so $w \Vdash_{\mathcal{K}_{t}} A_{1} \vee A_{2}$. If one of the latter two cases hold, then by I.H. $w \Vdash_{\mathcal{K}_{t}} A_{i}$ for one of $i \in\{1,2\}$; so $w \Vdash_{\mathcal{K}_{t}} A_{1} \vee A_{2}$. Hence we conclude $w \Vdash_{\mathcal{K} t} A_{1} \vee A_{2}$ for all $w \in W_{K}$, i.e. $\mathcal{M}_{\mathcal{K}}^{t} \vDash_{\mathcal{K} t} A_{1} \vee A_{2}$. For the converse direction, assume $\mathcal{M}_{\mathcal{K}}^{t} \vDash_{\mathcal{K} t} A_{1} \vee A_{2}$ and let $\alpha=\langle w, \ldots\rangle \in\langle\rangle$. Then since $w \Vdash_{\mathcal{K}_{t}} A_{1}$ or $w \Vdash_{\mathcal{K} t} A_{2},\langle w\rangle \Vdash_{\mathcal{B}} A_{1}$ or $\langle w\rangle \Vdash_{\mathcal{B}} A_{2}$ by I.H.. Thus $\forall \alpha \in\langle\rangle \exists m\left(\bar{\alpha} m \Vdash_{\mathcal{B}} A_{1}\right.$ or $\left.\bar{\alpha} m \Vdash_{\mathcal{B}} A_{2}\right)$. Hence \langle\rangle$\Vdash_{\mathcal{B}} A_{1} \vee A_{2}$.

For 2. If $\left\langle b_{0}, \ldots, b_{n}\right\rangle \Vdash_{\mathcal{B}} A_{1} \vee A_{2}$, then for all $\alpha \in\left\langle b_{0}, \ldots, b_{n}\right\rangle$ there exists $m$ s.t. $\bar{\alpha} m \Vdash_{\mathcal{B}} A_{1}$ or $\bar{\alpha} m \Vdash_{\mathcal{B}} A_{2}$. As $\left\langle b_{0}, \ldots, b_{n}, b_{n}, \ldots\right\rangle \in$ $\left\langle b_{0}, \ldots, b_{n}\right\rangle$, we have, for $i \in\{1,2\}$, either \langle\rangle$\Vdash_{\mathcal{B}} A_{i},\left\langle b_{0}, \ldots, b_{l}\right\rangle \Vdash_{\mathcal{B}} A_{i}$ for $l \leq n$, or $\left\langle b_{0}, \ldots, b_{n}, b_{n}, \ldots, b_{n}\right\rangle \Vdash_{\mathcal{B}} A_{i}$. In each case $b_{n} \Vdash_{\mathcal{K}_{t}} A_{i}$ by I.H.; so $b_{n} \Vdash_{\mathcal{K}_{t}} A_{1} \vee A_{2}$. Conversely, if $b_{n} \Vdash_{\mathcal{K} t} A_{1} \vee A_{2}$, then $b_{n} \Vdash_{\mathcal{K}_{t}} A_{1}$ or $b_{n} \Vdash_{\mathcal{K}_{t}} A_{2}$. So by I.H. $\left\langle b_{0}, \ldots, b_{n}\right\rangle \Vdash_{\mathcal{B}} A_{1}$ or $\left\langle b_{0}, \ldots, b_{n}\right\rangle \Vdash_{\mathcal{B}} A_{2}$. Hence immediately $\forall \alpha \in\left\langle b_{0}, \ldots, b_{n}\right\rangle \exists m\left(\bar{\alpha} m \Vdash_{\mathcal{B}} A_{1}\right.$ or $\left.\bar{\alpha} m \Vdash_{\mathcal{B}} A_{2}\right)$, i.e. $\left\langle b_{0}, \ldots, b_{n}\right\rangle \vdash_{\mathcal{B}} A_{1} \vee A_{2}$.

If $A \equiv A_{1} \rightarrow A_{2}$, then for 1 ., suppose \langle\rangle$\vdash_{\mathcal{B}} A_{1} \rightarrow A_{2}$. Let $w \in$ $W_{K}$ and $w^{\prime} \geq w$. If $w^{\prime} \Vdash_{\mathcal{K} t} A_{1}$, then $\left\langle w^{\prime}\right\rangle \Vdash_{\mathcal{B}} A_{1}$ by I.H.. So $\left\langle w^{\prime}\right\rangle \Vdash_{\mathcal{B}} A_{2}$ and thus $w^{\prime} \Vdash_{\mathcal{K} t} A_{2}$. Consequently $w \Vdash_{\mathcal{K} t} A_{1} \rightarrow A_{2}$ and so $\mathcal{M}_{\mathcal{K}}^{t} \vDash_{\mathcal{K} t} A_{1} \rightarrow A_{2}$. Conversely, suppose $\mathcal{M}_{\mathcal{K}}^{t} \vDash_{\mathcal{K} t} A_{1} \rightarrow A_{2}$. Let $\left\langle b_{0}, \ldots, b_{n}\right\rangle \Vdash_{\mathcal{B}} A_{1}$. If $n=-1$, then by I.H. $\mathcal{M}_{\mathcal{K}}^{t} \vDash_{\mathcal{K} t} A_{1}$, so $\mathcal{M}_{\mathcal{K}}^{t} \vDash_{\mathcal{K}_{t}} A_{2}$. Hence $\left\langle b_{0}, \ldots, b_{n}\right\rangle \Vdash_{\mathcal{B}} A_{2}$ again by I.H.. If $n>-1$, then $b_{n} \Vdash_{\mathcal{K}_{t}} A_{1}$, so $b_{n} \Vdash_{\mathcal{K} t} A_{2}$. Hence $\left\langle b_{0}, \ldots, b_{n}\right\rangle \Vdash_{\mathcal{B}} A_{2}$. Thus \langle\rangle$\Vdash_{\mathcal{B}} A_{1} \rightarrow A_{2}$. 
For 2., suppose $\left\langle b_{0}, \ldots, b_{n}\right\rangle \Vdash_{\mathcal{B}} A_{1} \rightarrow A_{2}$ and let $b_{n^{\prime}} \geq b_{n}$. If $b_{n^{\prime}} \Vdash_{\mathcal{K}_{t}}$ $A_{1}$, then by I.H. $\left\langle b_{0}, \ldots, b_{n}, b_{n^{\prime}}\right\rangle \Vdash_{\mathcal{B}} A_{1}$; so $\left\langle b_{0}, \ldots, b_{n}, b_{n^{\prime}}\right\rangle \Vdash_{\mathcal{B}} A_{2}$. Thus $b_{n^{\prime}} \Vdash_{\mathcal{K} t} A_{2}$. Hence $b_{n} \Vdash_{\mathcal{K}_{t}} A_{1} \rightarrow A_{2}$. Conversely, suppose $b_{n} \Vdash_{\mathcal{K}_{t}} A_{1} \rightarrow A_{2}$. Assume $\left\langle b_{0}, \ldots, b_{n}, \ldots, b_{n^{\prime}}\right\rangle \Vdash_{\mathcal{B}} A_{1}$. Then $b_{n} \leq b_{n^{\prime}}$ and $b_{n^{\prime}} \Vdash_{\mathcal{K} t} A_{1}$. So $b_{n^{\prime}} \Vdash_{\mathcal{K}_{t}} A_{2}$. Thus $\left\langle b_{0}, \ldots, b_{n}, \ldots, b_{n^{\prime}}\right\rangle \Vdash_{\mathcal{B}} A_{2}$. Therefore $\left\langle b_{0}, \ldots, b_{n}\right\rangle \Vdash_{\mathcal{B}} A_{1} \rightarrow A_{2}$.

If $A \equiv \sim A_{1}$, then for 1 ., suppose \langle\rangle$\Vdash_{\mathcal{B}} \sim A_{1}$. Then \langle\rangle$\nVdash_{\mathcal{B}} A_{1}$. So $\mathcal{M}_{\mathcal{K}}^{t} \not \nvdash_{\mathcal{K} t} A_{1}$ by I.H.. Hence $w \nVdash_{\mathcal{K} t} A_{1}$ for some $w \in W_{K}$. Thus $u \Vdash_{\mathcal{K} t} \sim A$ for all $u \in W_{K}$. Thus $\mathcal{M}_{\mathcal{K}}^{t} \vDash_{\mathcal{K} t} \sim A$. Conversely, suppose $\mathcal{M}_{\mathcal{K}}^{t} \vDash_{\mathcal{K} t} \sim A$. Take $w \in W_{K}$. Then $w \Vdash_{\mathcal{K} t} \sim A$, so $u \nVdash_{\mathcal{K}_{t}} A$ for some $u \in W_{K}$. Hence $\mathcal{M}_{\mathcal{K}}^{t} \nvdash_{\mathcal{K} t} A$, so \langle\rangle$\nVdash_{\mathcal{B}} A$ by I.H.. Therefore \langle\rangle$\Vdash_{\mathcal{B}} \sim A$. For 2., suppose $\left\langle b_{0}, \ldots, b_{n}\right\rangle \Vdash_{\mathcal{B}} \sim A$. Then \langle\rangle$\nVdash_{\mathcal{B}} A$. So $\mathcal{M}_{\mathcal{K}}^{t} \nvdash_{\mathcal{K} t}$ $A$. Hence for some $w \in W_{K}, w \nVdash_{\mathcal{K} t} A$. Therefore $b_{n} \Vdash_{\mathcal{K} t} \sim A$. Conversely, if $b_{n} \Vdash_{\mathcal{K}_{t}} \sim A$, then $w \nVdash_{\mathcal{K}_{t}} A$ for some $w \in W_{K}$. By I.H. $\langle w\rangle \nVdash_{\mathcal{B}} A$. Thus \langle\rangle$\nVdash_{\mathcal{B}} A$. Therefore $\left\langle b_{0}, \ldots, b_{n}\right\rangle \Vdash_{\mathcal{B}} \sim A$.

Theorem 4.9 (soundness and weak completeness of $\mathbf{T C C}_{\omega}$ with Beth semantics). $\vdash_{t} A$ if and only if $\vDash_{\mathcal{B}} A$.

Proof: We first show the soundness by induction on the depth of deductions. We check $[\mathrm{Ax} 9],[\mathrm{Ax} 10]$ and $[\mathrm{RC}]$. Let $\mathcal{M}_{\mathcal{B}}=\left(W_{B}, \preceq, \mathcal{V}_{B}\right)$ be a Beth model. By monotonicity, it suffices to check the root. For [Ax9], either \langle\rangle$\Vdash_{\mathcal{B}} A$ or \langle\rangle$\nVdash_{\mathcal{B}} A$. If the latter, \langle\rangle$\Vdash_{\mathcal{B}} \sim A$. So in either case, \langle\rangle$\Vdash_{\mathcal{B}} A \vee \sim A$. For [Ax10], if $b \Vdash_{\mathcal{B}} \sim A$ for $b \succeq\langle\rangle$, then if $b^{\prime} \Vdash_{\mathcal{B}} \sim \sim A$ for $b^{\prime} \succeq b$, then \langle\rangle$\nVdash_{\mathcal{B}} \sim A$ and \langle\rangle$\nVdash_{\mathcal{B}} A$. But the former implies \langle\rangle$\Vdash_{\mathcal{B}} A$, a contradiction. Therefore $b^{\prime} \Vdash_{\mathcal{B}} B$; so \langle\rangle$\Vdash_{\mathcal{B}} \sim A \rightarrow(\sim \sim A \rightarrow B)$. For [RC], by I.H., $\vDash_{\mathcal{B}} A \rightarrow B$ and in particular, $\mathcal{M}_{\mathcal{B}} \vDash_{\mathcal{B}} A \rightarrow B$. If for $b \succeq\langle\rangle$ we have $b \Vdash_{\mathcal{B}} \sim B$, then \langle\rangle$\nVdash_{\mathcal{B}} B$. Now if \langle\rangle$\Vdash_{\mathcal{B}} A$, then as \langle\rangle$\Vdash_{\mathcal{B}} A \rightarrow B,\langle\rangle \Vdash_{\mathcal{B}} B$, a contradiction. Thus \langle\rangle$\nVdash_{\mathcal{B}} A$; hence $b \Vdash_{\mathcal{B}} \sim A$. So \langle\rangle$\Vdash_{\mathcal{B}} \sim B \rightarrow \sim A$.

The completeness follows from the previous lemma and the Kripke completeness of $\mathbf{T} \mathbf{C C}_{\omega}$ [7, Theorem 4.5].

\subsection{Classical Logic and $\mathbf{T C C}_{\omega}$}

The fact that Kripke and Beth semantics differ on the forcing of disjunction is well-reflected in the following translation of classical logic (CPC) into $\mathbf{T C C}_{\omega}$. 
Definition 4.10 (CPC). CPC is defined by Axioms [Ax1]-[Ax9] and $\sim A \rightarrow(A \rightarrow B)\left(\left[\mathrm{Ax} 10^{\prime}\right]\right)$, plus the rule $[\mathrm{MP}]$.

We denote the derivability in $\mathbf{C P C}$ by $\vdash_{C L}$

Definition $4.11\left(()^{t}\right)$. We inductively define ()$^{t}$ to be a mapping between formulae in $\mathcal{L}$.

$$
\begin{aligned}
p^{t} & \equiv p \\
(A \wedge B)^{t} & \equiv A^{t} \wedge B^{t} . \\
(A \vee B)^{t} & \equiv \sim \sim A^{t} \vee \sim \sim B^{t} . \\
(A \rightarrow B)^{t} & \equiv \sim \sim A^{t} \rightarrow \sim \sim B^{t} . \\
(\sim A)^{t} & \equiv \sim A^{t} .
\end{aligned}
$$

Beth-semantically speaking, ()$^{t}$ restricts our attention to the root world, when it comes to disjunction and implication. This is related to the connection between empirical negation (of $\mathbf{I P C}^{\sim}$ ) and classical negation, as observed in [3] and [4]. A new point for $\mathbf{T} \mathbf{C C} \omega$ is that the restriction applies not only to implication but also to disjunction. This corresponds to the fact that in Beth semantics, both disjunction and implication look at other worlds, whereas in Kripke semantics, only the latter does so.

In the following, we make a heavy use of easily checkable equivalences in Beth semantics.

- $b \Vdash_{\mathcal{B}} \sim \sim A \Longleftrightarrow\langle\rangle \Vdash_{\mathcal{B}} A$.

- $b \Vdash_{\mathcal{B}} \sim \sim A \vee \sim \sim B \Longleftrightarrow\langle\rangle \Vdash_{\mathcal{B}} A$ or \langle\rangle$\Vdash_{\mathcal{B}} B$.

- $b \Vdash_{\mathcal{B}} \sim \sim A \rightarrow \sim \sim B \Longleftrightarrow\langle\rangle \Vdash_{\mathcal{B}} A$ implies \langle\rangle$\Vdash_{\mathcal{B}} B$.

Let us use the notation $\Gamma^{t}:=\left\{B^{t}: B \in \Gamma\right\}$. We shall henceforth abbreviate $\sim \sim A$ as $\approx A$. Metalinguistic 'implies' $(\Rightarrow)$ should not be confused with $\rightarrow$ in the proof below.

Proposition 4.12 (faithful embedding of CPC into $\mathbf{T C C}_{\omega}$ ). $\Gamma \vdash_{C L} A$ if and only if $\Gamma^{t} \vdash_{t} A^{t}$.

Proof: The left-to-right direction is shown by induction on the depth of deductions. If $A$ is an assumption, then correspondingly $A^{t} \in \Gamma^{t}$. 
If $A$ is an axiom, we exemplify by the case for the axiom $(A \rightarrow C) \rightarrow$ $((B \rightarrow C) \rightarrow(A \vee B \rightarrow C)) .((A \rightarrow C) \rightarrow((B \rightarrow C) \rightarrow(A \vee B \rightarrow C)))^{t}$ is

$$
\approx\left(\approx A^{t} \rightarrow \approx C^{t}\right) \rightarrow \approx\left(\approx\left(\approx B^{t} \rightarrow \approx C^{t}\right) \rightarrow \approx\left(\approx\left(\approx A^{t} \vee \approx B^{t}\right) \rightarrow \approx C^{t}\right)\right) .
$$

Using Beth completeness, it is sufficient to show,

$b \Vdash_{\mathcal{B}} \approx\left(\approx A^{t} \rightarrow \approx C^{t}\right) \rightarrow \approx\left(\approx\left(\approx B^{t} \rightarrow \approx C^{t}\right) \rightarrow \approx\left(\approx\left(\approx A^{t} \vee \approx B^{t} \rightarrow \approx C^{t}\right)\right)\right)$

holds for any $b$ in an arbitrary Beth model. This is equivalent to

$$
\begin{aligned}
&\langle\rangle \Vdash_{\mathcal{B}} \approx A^{t} \rightarrow \approx C^{t} \\
& \text { implies }\langle\rangle \vdash_{\mathcal{B}} \approx\left(\approx B^{t} \rightarrow \approx C^{t}\right) \rightarrow \approx\left(\approx\left(\approx A^{t} \vee \approx B^{t}\right) \rightarrow \approx C^{t}\right)
\end{aligned}
$$

by one of the above equivalences; this is further equivalent to

$$
\begin{gathered}
\langle\rangle \Vdash_{\mathcal{B}} A^{t} \Rightarrow\langle\rangle \Vdash_{\mathcal{B}} C^{t} \\
\text { implies }\left(\langle\rangle \Vdash_{\mathcal{B}} \approx B^{t} \rightarrow \approx C^{t}\right) \Rightarrow\left(\langle\rangle \Vdash_{\mathcal{B}} \approx\left(\approx\left(\approx A^{t} \vee \approx B^{t}\right) \rightarrow \approx C^{t}\right)\right)
\end{gathered}
$$

and to

$$
\langle\rangle \Vdash_{\mathcal{B}} A^{t} \Rightarrow\langle\rangle \Vdash_{\mathcal{B}} C^{t}
$$

implies $\left(\langle\rangle \Vdash_{\mathcal{B}} B^{t} \Rightarrow\langle\rangle \Vdash_{\mathcal{B}} C^{t}\right) \Rightarrow\left(\langle\rangle \Vdash_{\mathcal{B}} \approx A^{t} \vee \approx B^{t} \Rightarrow\langle\rangle \Vdash_{\mathcal{B}} C^{t}\right)$

and to

$$
\begin{array}{r}
\left(\langle\rangle \Vdash_{\mathcal{B}} A^{t} \Rightarrow\langle\rangle \Vdash_{\mathcal{B}} C^{t}\right) \text { and }\left(\langle\rangle \Vdash_{\mathcal{B}} B^{t} \Rightarrow\langle\rangle \Vdash_{\mathcal{B}} C^{t}\right) \\
\text { implies } \left.\left(\left(\langle\rangle \Vdash_{\mathcal{B}} A^{t} \text { or }\langle\rangle \Vdash_{\mathcal{B}} B^{t}\right) \Rightarrow\langle\rangle \Vdash_{\mathcal{B}} C^{t}\right)\right)
\end{array}
$$

and this holds. Here, if it were the case that $(A \vee B)^{t} \equiv\left(A^{t} \vee B^{t}\right)$, then we would get \langle\rangle$\Vdash_{\mathcal{B}} A^{t} \vee B^{t}$ instead of \langle\rangle$\Vdash_{\mathcal{B}} \approx A^{t} \vee \approx B^{t}$, and the formula fails to hold.

If the deduction ends with an application of $[\mathrm{MP}] \frac{B \quad B \rightarrow A}{A}$, then by I.H., $\Gamma^{t} \vdash_{t} B^{t}$ and $\Gamma^{t} \vdash_{t} \sim \sim B^{t} \rightarrow \sim \sim A^{t}$. In [4, Lemma 2.8] the rule $\frac{A}{\sim \sim A}[\mathrm{RD}]$ is shown to be derivable from $[\mathrm{RC}]$ in $\mathbf{I P C}^{\sim}$. The proof appeals to [RP] only non-essentially (it is used to derive $\sim \sim A \rightarrow A$, which is obtainable from [Ax9] and [Ax10] alone), and so [RD] is also derivable in $\mathbf{T C C}_{\omega}$. Thus we obtain $\Gamma^{t} \vdash_{t} \sim \sim B^{t}$. So by [MP], $\Gamma^{t} \vdash_{t} \sim \sim A^{t}$; hence $\Gamma^{t} \vdash_{t} A^{t}$ by double negation elimination. 
The right-to-left direction follows from the easily noticeable equivalence that $\vdash_{C L} A \leftrightarrow A^{t}$.

Before moving on, we shall mention that there exists another reading of the negation in the Beth semantics for $\mathbf{T} \mathbf{C C} \omega$. Because the models are rooted, for any $b, \exists b^{\prime} \leq b\left(b^{\prime} \nVdash A\right) \Leftrightarrow\langle\rangle \nVdash A$. From this viewpoint the negation of $\mathbf{T} \mathbf{C C}_{\omega}$ can be understood as co-negation as well. For Kripke semantics, the logic of co-negation is the logic daC of Priest [11]. A Hilbert-style axiomatisation of $\mathbf{d a C}$ was first formulated by Castiglioni et al. [2]. This axiomatisation is obtained from that of $\mathbf{I P C}^{\sim}$ by removing [Ax10]. If we further replace [RP] with [RC], and add an axiom $\sim A \rightarrow A$ (a theorem of daC), we obtain the logic $\mathbf{C C}_{\omega}$ of Sylvan [15]. Note $\mathbf{C C}_{\omega}$ can be strengthened to $\mathbf{T C C}_{\omega}$ by adding [Ax10] and dropping $\sim \sim A \rightarrow A$, which becomes redundant.

\section{Eliminating $[\mathrm{RP}]$}

The last section made clear that the negations of $\mathbf{I P C}{ }^{\sim}$ and $\mathbf{T} \mathbf{C C}_{\omega}$ are characterised by the same valuation, but with respect to different semantics: Kripke and Beth. We may understand them as representing different types of experience, and thus different empirical negations. We can make an analogous remark for co-negation. This case is perhaps more interesting, for $\mathbf{T} \mathbf{C C} \omega$ and $\mathbf{d a C}$ are not comparable [10]. In any case, these curious effects of "same forcing-condition in two similar semantics" encourage a further analysis.

Proof-theoretically, however, there is an obstacle in comparing the logics, in that $\mathbf{T} \mathbf{C C}_{\omega}$ and $\mathbf{C C}_{\omega}$ employ the rule $[\mathrm{RC}]$, whereas $\mathbf{~ d a C}$ and $\mathbf{I P C} \mathbf{C}^{\sim}$ employ the stronger $[\mathrm{RP}]$.

We would like, therefore, to have a new axiomatisation of IPC ${ }^{\sim}$ and daC with $[\mathrm{RC}]$, rather than $[\mathrm{RP}]$. We can expect such conversion would allow us to analyse and understand the logics from a more unified perspective.

We shall start such an attempt with IPC $^{\sim}$, using a provable formula of IPC $^{\sim},(\sim A \wedge \sim B) \rightarrow \sim(A \vee B)$ [4, Proposition 2.14].

Proposition 5.1. The addition of $(\sim A \wedge \sim B) \rightarrow \sim(A \vee B)$ to $\mathbf{T C C}_{\omega}$ derives $[\mathrm{RP}]$. 
Proof: In $\mathbf{T C C}_{\omega}$, assuming $(A \vee B)$ we can derive $\sim \sim(A \vee B)$ by [RD]. So we have $\sim B \rightarrow(\sim A \rightarrow \sim \sim(A \vee B))$. Also we infer from $\sim B \rightarrow(\sim A \rightarrow$ $(\sim A \wedge \sim B))$ and $(\sim A \wedge \sim B) \rightarrow \sim(A \vee B)$ that $\sim B \rightarrow(\sim A \rightarrow \sim(A \vee B))$. Thus $\sim B \rightarrow(\sim A \rightarrow(\sim(A \vee B) \wedge \sim \sim(A \vee B)))$. Also by [Ax10], $\sim(A \vee B) \rightarrow$ $(\sim \sim(A \vee B) \rightarrow B)$. Combine the two and we obtain $\sim B \rightarrow(\sim A \rightarrow B)$. Then as $B \rightarrow(\sim A \rightarrow B)$ follows from [Ax1], and $B \vee \sim B$ follows from [Ax9], we conclude $\sim A \rightarrow B$.

Hence we have obtained an alternative axiomatisation of IPC $^{\sim}$ with $[\mathrm{RC}]$.

It is stated in [4] that $\mathbf{T} \mathbf{C C}_{\omega}$ is a strict subsystem of $\mathbf{I P C} \mathbf{C}^{\sim}$, but no specific example is shown. As a side remark, we can use $(\sim A \wedge \sim B) \rightarrow$ $\sim(A \vee B)$ to observe the following.

Proposition 5.2. $(\sim A \wedge \sim B) \rightarrow \sim(A \vee B)$ is underivable in $\mathbf{T C C}_{\omega}$.

Proof: We prove it via Beth completeness. Let $\mathcal{F}_{\mathcal{B}}=(W, \preceq)$ be the set of finite binary sequences ordered by the initial segment relation. Let $\mathcal{M}_{\mathcal{B}}=\left(\mathcal{F}_{\mathcal{B}}, \mathcal{V}\right)$ be a model such that $b \in \mathcal{V}(p) \Leftrightarrow\langle 0\rangle \preceq b$ and $b \in \mathcal{V}(q) \Leftrightarrow$ $\langle 1\rangle \preceq b$. Then it is straightforward to see that this assignment is covering: e.g. if $\forall \alpha \in b \exists m\left(\bar{\alpha} m \Vdash_{\mathcal{B}} p\right)$, then clearly $\langle 0\rangle \preceq b$. Now $\mathcal{M}_{\mathcal{B}},\langle\rangle \nVdash_{\mathcal{B}} p$ and $\mathcal{M}_{\mathcal{B}},\langle\rangle \nVdash_{\mathcal{B}} q$, so $\mathcal{M}_{\mathcal{B}},\langle\rangle \Vdash_{\mathcal{B}} \sim p \wedge \sim q$; but since $\forall \alpha \in\langle\rangle\left(\bar{\alpha} 1 \Vdash_{\mathcal{B}} p\right.$ or $\bar{\alpha} 1 \Vdash_{\mathcal{B}}$ $q$ ), we have $\mathcal{M}_{\mathcal{B}},\langle\rangle \Vdash_{\mathcal{B}} p \vee q$, i.e. $\mathcal{M}_{\mathcal{B}},\langle\rangle \nVdash_{\mathcal{B}} \sim(p \vee q)$. Therefore $\mathcal{M}_{\mathcal{B}},\langle\rangle \nVdash_{\mathcal{B}}$ $(\sim p \wedge \sim q) \rightarrow \sim(p \vee q)$.

COROLlary 5.3 (failure of soundness for $\mathbf{I P C}^{\sim}$ with all Beth models). $\vdash \sim A \nRightarrow \vDash_{\mathcal{B}} A$.

Proof: Otherwise $\vdash_{\sim} A \Rightarrow \vDash_{\mathcal{B}} A \Leftrightarrow \vdash_{t} A$, which is absurd.

Ferguson [6, Theorem 2.3] gives the frame property of $(\sim A \wedge \sim B) \rightarrow$ $\sim(A \vee B)$ with respect to daC. We just mention a quite similar observation can be made for the Kripke models for $\mathbf{C C}_{\omega}$.

Definition 5.4 (Semantics of $\mathbf{C C}_{\omega}$ ). A Kripke frame $\mathcal{F}_{\mathcal{K}}^{c}$ for $\mathbf{C C}_{\omega}$ is a triple $(W, \leq, S)$, where $S \subset W \times W$ is a reflexive and symmetric (accessibility) relation such that $u \leq v$ and $u S w$ implies $v S w$, i.e. $S$ is upward 
closed. A Kripke model $\mathcal{M}_{\mathcal{K}}^{c}$ for $\mathbf{C C}_{\omega}$ is defined as usual, except for the forcing condition $\left(\Vdash_{\mathcal{K}_{c}}\right)$ of negation, which is

$$
\mathcal{M}_{\mathcal{K}}^{c}, w \Vdash_{\mathcal{K}_{c}} \sim A \Longleftrightarrow \mathcal{M}_{\mathcal{K}}^{c}, w^{\prime} \nVdash_{\mathcal{K}_{c}} A \text { for some } w^{\prime} \text { such that } w S w^{\prime} .
$$

Note if $S=W \times W$, then a $\mathbf{C C}_{\omega}$-frame (model) is a $\mathbf{T} \mathbf{C C}_{\omega}$-frame (model) [7]. Indeed, what is shown in [7] is that $\mathbf{T} \mathbf{C C}_{\omega}$ is sound and complete with the class of $\mathbf{C C}_{\omega}$-frames where $S$ is transitive, and in particular the frames with $S=W \times W$ is sufficient for this. We shall occasionally denote $u S v$ also by $v S^{-1} u$. As $S$ is symmetric in $\mathbf{C C}_{\omega}$, this distinction is not quite necessary. This however clarifies appeals to symmetry in proofs, which becomes significant in a broader context.

Proposition 5.5. Let $\mathcal{F}_{\mathcal{K}}^{c}$ be a $\mathbf{C C}_{\omega}$-frame. Then the following conditions are equivalent:

(i) $\mathcal{F}_{\mathcal{K}}^{c} \vDash_{\mathcal{K}_{c}}(\sim A \wedge \sim B) \rightarrow \sim(A \vee B)$ for all $A, B$.

(ii) $\mathcal{F}_{\mathcal{K}}^{c}$ satisfies $\forall u, v, w\left(u S v\right.$ and $u S w$ implies $\exists x S^{-1} u(v \geq x$ and $w \geq x)$.

Proof: We shall first see (i) implies (ii). Suppose $u S v$ and $u S w$. Let $\mathcal{V}(p)=\{x: v \nsupseteq x\}$ and $\mathcal{V}(q)=\{x: w \nsupseteq x\}$. Now if $w \in \mathcal{V}(p)$ and $x^{\prime} \geq x$, then $v \geq x^{\prime}$ implies $v \geq x$, a contradiction. So $v \nsucceq x^{\prime}$, and thus $x^{\prime} \in \mathcal{V}(p)$. Hence $\mathcal{V}(p)$ is upward closed. Similarly $\mathcal{V}(q)$ is upward closed. Now since $v \geq v$ and $w \geq w, v \nVdash_{\mathcal{K}_{c}} p$ and $w \nVdash_{\mathcal{K}_{c}} q$. So $u \Vdash_{\mathcal{K}_{c}} \sim p \wedge \sim q$. Hence by assumption $u \Vdash_{\mathcal{K}_{c}} \sim(p \vee q)$. So there is an $x S^{-1} u$ such that $x \nVdash_{\mathcal{K}_{c}} p$ (i.e. $v \geq x$ ) and $x \nVdash_{\mathcal{K}_{c}} q$ (i.e. $w \geq x$ ), as we desired.

Next we shall see (ii) implies (i). Assume $\mathcal{F}_{\mathcal{K}}^{c}$ satisfies (ii) and $\mathcal{V}, u_{0}$ be arbitrary. If $\left(\mathcal{F}_{\mathcal{K}}^{c}, \mathcal{V}\right), u \Vdash_{\mathcal{K}_{c}} \sim A \wedge \sim B$ for $u \geq u_{0}$, then there are $v S^{-1} u$ and $w S^{-1} u$ such that $v \nVdash_{\mathcal{K}_{c}} A$ and $w \nVdash_{\mathcal{K}_{c}} B$. By (ii), there is $x S^{-1} u$ such that $v \geq x$ and $w \geq x$. Now $x \nVdash_{\mathcal{K}_{c}} A \vee B$. Hence $u \Vdash_{\mathcal{K}_{c}} \sim(A \vee B)$. So $\left(\mathcal{F}_{\mathcal{K}}^{c}, \mathcal{V}\right), u_{0} \Vdash_{\mathcal{K}_{c}}(\sim A \wedge \sim B) \rightarrow \sim(A \vee B)$. Since $w$ and $\mathcal{V}$ are arbitrary, $\mathcal{F}_{\mathcal{K}}^{c} \vDash_{\mathcal{K}_{c}}(\sim A \wedge \sim B) \rightarrow \sim(A \vee B)$.

Given a Kripke frame for $\mathbf{I P C}{ }^{\sim}$, we can regard it as a frame of $\mathbf{T} \mathbf{C C}_{\omega}$ with $S=W \times W$; i.e. there is an embedding. Then it is immediately seen that such a frame satisfies the above condition, because it is rooted. This means the class of Kripke frames for $\mathbf{T} \mathbf{C C} \omega$ satisfying the above condition is complete with respect to $\mathbf{I P C}^{\sim}$, for if a formula is validated by each such frame, then it must be validated by each frame of $\mathbf{I P C}^{\sim}$. 
Next we consider daC. The formula $\sim A \wedge \sim B \rightarrow \sim(A \vee B)$ used for IPC $^{\sim}$ cannot be used for daC, because it is not a theorem of daC [9, Table 3]. We instead have to look at another formula $\sim(\sim(A \vee B) \vee A) \rightarrow B$.

Proposition 5.6. $\mathbf{C C}_{\omega}+\sim(\sim(A \vee B) \vee A) \rightarrow B=$ daC.

Proof: It has been observed in [9, Theorem 3.13] that $\sim(\sim(A \vee B) \vee A) \rightarrow$ $B$ is a theorem of daC. So we only have to check $[\mathrm{RP}]$ is admissible in $\mathbf{C C}_{\omega}+\sim(\sim(A \vee B) \vee A) \rightarrow B$. We first note $\frac{A}{\sim A \rightarrow B}$ is derivable in $\mathbf{C C}_{\omega}$ by the same argument as in [10, Theorem 4.3]. Assuming $A \vee B$ is derivable, from this we see $\sim(A \vee B) \rightarrow A$ is derivable. By [Ax8], we infer $(\sim(A \vee B) \vee A) \rightarrow A$, and then by $[\mathrm{RC}], \sim A \rightarrow \sim(\sim(A \vee B) \vee A)$. On the other hand, $\sim(\sim(A \vee B) \vee A) \rightarrow B$ is the added axiom. Thus we conclude $\sim A \rightarrow B$.

$\sim(\sim(A \vee B) \vee A) \rightarrow B$ is used in [9, theorem 3.13] to establish that daC strictly contains another logic daC', axiomatised by replacing $[\mathrm{RP}]$ with a weaker rule $\frac{A \vee \sim B}{\sim A \rightarrow \sim B}[\mathrm{wRP}]$. We shall note $[\mathrm{wRP}]$ in $\mathbf{d a C}^{\prime}$ is similarly reducible to an axiom $\sim(\sim(A \vee \sim B) \vee A) \rightarrow \sim B$.

Proposition 5.7. $\mathbf{C C}_{\omega}+\sim(\sim(A \vee \sim B) \vee A) \rightarrow \sim B=\mathbf{d a C}^{\prime}$

Proof: It has been observed in [10, Lemma 3.2] that $\sim(\sim(A \vee \sim B) \vee$ $A) \rightarrow \sim B$ is a theorem of $\mathbf{d a C}^{\prime}$. So we only have to check [wRP] is admissible in $\mathbf{C C}_{\omega}+\sim(\sim(A \vee \sim B) \vee A) \rightarrow \sim B$. This is proved as in the previous proposition, except that we infer $\sim A \rightarrow \sim(\sim(A \vee \sim B) \vee A)$ and $\sim(\sim(A \vee \sim B) \vee A) \rightarrow \sim B$ to conclude $\sim A \rightarrow \sim B$

Next, we turn our attention to the semantic side. Our goal will be to establish a connection between the Kripke semantics of $\mathbf{C C}_{\omega}$ and daC. For this we shall first consider the frame condition for $\sim(\sim(A \vee B) \vee A) \rightarrow B$.

Proposition 5.8. Let $\mathcal{F}_{\mathcal{K}}^{c}$ be a $\mathbf{C C}_{\omega}$-frame. Then the following conditions are equivalent:

(i) $\mathcal{F}_{\mathcal{K}}^{c} \vDash_{\mathcal{K}_{c}} \sim(\sim(A \vee B) \vee A) \rightarrow B$ for all $A, B$.

(ii) $\mathcal{F}_{\mathcal{K}}^{c}$ satisfies $\forall u, v\left(u S v \rightarrow \exists w S^{-1} v(w \leq u\right.$ and $\left.w \leq v)\right)$.

Proof: We shall first see (i) implies (ii). We shall show the contrapositive. So suppose for some $u$ and $v, u S v$ holds but $\neg \exists w S^{-1} v(w \leq u$ and $w \leq$ 
$v)$. Choose $\mathcal{V}$ s.t. $\mathcal{V}(p)=\{w: w \not \leq v\}$ and $\mathcal{V}(q)=\{w: w \not \leq u\}$. It is straightforward to see $\mathcal{V}(p)$ and $\mathcal{V}(q)$ are upward closed. Now since $\forall w S^{-1} v(w \not \leq u$ or $w \not \leq v)$, we have $\forall w S^{-1} v\left(w \Vdash_{\mathcal{K}_{c}} p\right.$ or $\left.w \Vdash_{\mathcal{K}_{c}} q\right)$. So $v \nVdash_{\mathcal{K}_{c}} \sim(p \vee q)$. In addition, $v \leq v$ means $v \nVdash_{\mathcal{K}_{c}} p$. Thus $u \Vdash_{\mathcal{K}_{c}} \sim(\sim(p \vee$ $q) \vee p)$. On the other hand, $u \leq u$ implies $u \nVdash_{\mathcal{K}_{c}} q$. Thus $u \nVdash_{\mathcal{K}_{c}} \sim(\sim(p \vee$ q) $\vee p) \rightarrow q$. Therefore $\mathcal{F}_{\mathcal{K}}^{c} \not \nvdash_{\mathcal{K} c} \sim(\sim(p \vee q) \vee p) \rightarrow q$.

Next we shall see (ii) implies (i). Assume $\forall u, v\left(u S v \rightarrow \exists w S^{-1} v(w \leq\right.$ $u$ and $w \leq v)$ ). Let $\mathcal{V}$ and $u$ be arbitrary, and for $v \geq u$, suppose $\left(\mathcal{F}_{\mathcal{K}}^{c}, \mathcal{V}\right), v \Vdash_{\mathcal{K}_{c}} \sim(\sim(A \vee B) \vee A)$. Then for some $w S^{-1} v, w \nVdash_{\mathcal{K}_{c}} \sim(A \vee B) \vee A$. Thus $w \nVdash_{\mathcal{K}_{c}} A$ and $\forall x S^{-1} w\left(x \Vdash_{\mathcal{K}_{c}} A \vee B\right)$. Now by assumption, from $v S w$ we infer $\exists y S^{-1} w(y \leq v$ and $y \leq w)$. From our observation above, we know $y \Vdash_{\mathcal{K}_{c}} A \vee B$. If $y \Vdash_{\mathcal{K}_{c}} A$, then $y \leq w$ implies $w \Vdash_{\mathcal{K}_{c}} A$, a contradiction. So $y \Vdash_{\mathcal{K}_{c}} B$, which with $y \leq v$ implies $v \Vdash_{\mathcal{K}_{c}} B$. Thus $\left(\mathcal{F}_{\mathcal{K}}^{c}, \mathcal{V}\right), u \Vdash_{\mathcal{K}_{c}} \sim(\sim(A \vee B) \vee A) \rightarrow B$. Since $\mathcal{V}$ and $u$ are arbitrary, $\mathcal{F}_{\mathcal{K}}^{c} \vDash_{\mathcal{K}_{c}} \sim(\sim(A \vee B) \vee A) \rightarrow B$

Note that in the proof no appeal is made to neither the reflexivity nor symmetry of $S$. Thus we see the correspondence holds for a weaker setting of one of Došen's systems in [5, p.81-83] (under what he calls condensed frames). It has the same forcing condition, but the accessibility relation there is not assumed to be reflexive nor symmetric.

With the frame condition at hand, we can now translate back and forth the frames of $\mathbf{C} \mathbf{C}_{\omega}$ and $\mathbf{d a C}$.

Definition 5.9 (semantics of $\mathbf{d a C}$ ). A Kripke frame $\mathcal{F}_{\mathcal{K}}^{d}$ for daC is a pair $(W, \leq)$, and a Kripke model $\mathcal{M}_{\mathcal{K}}^{d}$ for daC is defined as usual, except for the forcing condition $\left(\vdash_{\mathcal{K}_{c}}\right)$ of negation, which is

$$
\mathcal{M}_{\mathcal{K}}^{d}, w \Vdash_{\mathcal{K} d} \sim A \Longleftrightarrow \mathcal{M}_{\mathcal{K}}^{d}, w^{\prime} \nVdash_{\mathcal{K} d} A \text { for some } w^{\prime} \leq w .
$$

Proposition 5.10.

(i) Let $\mathcal{F}_{\mathcal{K}}^{c}=(W, \leq, S)$ be a frame of $\mathbf{C C}_{\omega}$ satisfying $\forall u, v(u S v \rightarrow$ $\exists w S^{-1} v(w \leq u$ and $\left.w \leq v)\right)$. Define $\Phi\left(\mathcal{F}_{\mathcal{K}}^{c}\right)=(W, \leq)$. Then for any $\mathcal{V}$ and $w,\left(\mathcal{F}_{\mathcal{K}}^{c}, \mathcal{V}\right), w \Vdash_{\mathcal{K}_{c}} A \Leftrightarrow\left(\Phi\left(\mathcal{F}_{\mathcal{K}}^{c}\right), \mathcal{V}\right), w \Vdash_{\mathcal{K}_{d}} A$.

(ii) Let $\mathcal{F}_{\mathcal{K}}^{d}$ be a frame of daC. Define $S=\{(u, v): \exists w(w \leq u$ and $w \leq$ $v))\}$. and $\Psi\left(\mathcal{F}_{\mathcal{K}}^{d}\right)=(W, \leq, S)$. Then for any $\mathcal{V}$ and $w,\left(\mathcal{F}_{\mathcal{K}}^{d}, \mathcal{V}\right), w \Vdash_{\mathcal{K} d}$ $A \Leftrightarrow\left(\Psi\left(\mathcal{F}_{\mathcal{K}}^{d}\right), \mathcal{V}\right), w \Vdash_{\mathcal{K}_{c}} A$.

(iii) $\Psi=\Phi^{-1}$ for the above $\Phi$ and $\Psi$. 
Note the $S$ defined in (ii) is well-defined: it is easy to check it is reflexive, symmetric and satisfies $\forall u, v\left(u S v \rightarrow \exists w S^{-1} v(w \leq u\right.$ and $\left.w \leq v)\right)$.

Proof: In (i) and (ii), we only have to consider the case for negation.

For (i), if $\left(\mathcal{F}_{\mathcal{K}}^{c}, \mathcal{V}\right), w \Vdash_{\mathcal{K}_{c}} \sim A$, then for some $w^{\prime} S^{-1} w,\left(\mathcal{F}_{\mathcal{K}}^{c}, \mathcal{V}\right), w^{\prime} \nVdash_{\mathcal{K}_{c}} A$. By the frame condition, there is $x S^{-1} w$ such that $x \leq w$ and $x \leq w^{\prime}$. Because of the latter, $\left(\mathcal{F}_{\mathcal{K}}^{c}, \mathcal{V}\right), x \nVdash_{\mathcal{K} c} A$. By I.H., $\left(\Phi\left(\mathcal{F}_{\mathcal{K}}^{c}\right), \mathcal{V}\right), x \nVdash_{\mathcal{K} d}$ $A$. Since $x \leq w,\left(\Phi\left(\mathcal{F}_{\mathcal{K}}^{c}\right), \mathcal{V}\right), w \Vdash_{\mathcal{K}_{d}} \sim A$. For the converse direction, if $\left(\Phi\left(\mathcal{F}_{\mathcal{K}}^{c}\right), \mathcal{V}\right), w \Vdash_{\mathcal{K} d} \sim A$ then for some $w^{\prime} \leq w,\left(\Phi\left(\mathcal{F}_{\mathcal{K}}^{c}\right), \mathcal{V}\right), w^{\prime} \nVdash_{\mathcal{K}_{d}} A$. Вy I.H., $\left(\mathcal{F}_{\mathcal{K}}^{c}, \mathcal{V}\right), w^{\prime} \nVdash_{\mathcal{K}_{c}} A$. Here, since $w^{\prime} S w^{\prime}$ by reflexivity and $w^{\prime} \leq w$, we have $w^{\prime} S w$, so by symmetry $w S w^{\prime}$. Thus $\left(\mathcal{F}_{\mathcal{K}}^{c}, \mathcal{V}\right), w \Vdash_{\mathcal{K}_{c}} \sim A$.

For (ii), if $\left(\mathcal{F}_{\mathcal{K}}^{d}, \mathcal{V}\right), w \Vdash_{\mathcal{K} d} \sim A$, then for some $w^{\prime} \leq w,\left(\mathcal{F}_{\mathcal{K}}^{d}, \mathcal{V}\right), w^{\prime} \nVdash_{\mathcal{K} d}$ $A$. By I.H., $\left(\Psi\left(\mathcal{F}_{\mathcal{K}}^{d}\right), \mathcal{V}\right), w^{\prime} \nVdash_{\mathcal{K}_{c}} A$. Now as $w^{\prime} \leq w$ and $w^{\prime} S w^{\prime}, w S w^{\prime}$. So $\left(\Psi\left(\mathcal{F}_{\mathcal{K}}^{d}\right), \mathcal{V}\right), w \Vdash_{\mathcal{K}_{c}} \sim A$. For the converse direction, if $\left(\Psi\left(\mathcal{F}_{\mathcal{K}}^{d}\right), \mathcal{V}\right), w \Vdash_{\mathcal{K}_{c}}$ $\sim A$, then for some $w^{\prime} S^{-1} w,\left(\Psi\left(\mathcal{F}_{\mathcal{K}}^{d}\right), \mathcal{V}\right), w^{\prime} \nVdash_{\mathcal{K}_{c}} A$. Thus there is an $x$ such that $x \leq w$ and $x \leq w^{\prime}$. We have $\left(\Psi\left(\mathcal{F}_{\mathcal{K}}^{d}\right), \mathcal{V}\right), x \nVdash_{\mathcal{K}_{c}} A$ by the latter. By I.H., $\left(\mathcal{F}_{\mathcal{K}}^{d}, \mathcal{V}\right), x \nVdash_{\mathcal{K} d} A$. Therefore $\left(\mathcal{F}_{\mathcal{K}}^{d}, \mathcal{V}\right), w \Vdash_{\mathcal{K} d} \sim A$.

For (iii), it is immediate to see that $\Phi\left(\Psi\left(\mathcal{F}_{\mathcal{K}}^{d}\right)\right)=\mathcal{F}_{\mathcal{K}}^{d}$, as the mappings do not alter $(W, \leq)$. As for $\Psi\left(\Phi\left(\mathcal{F}_{\mathcal{K}}^{c}\right)\right)=\mathcal{F}_{\mathcal{K}}^{c}$, we need to check the original $S$ in $\mathcal{F}_{\mathcal{K}}^{c}$ and the defined $S^{\prime}$ in $\Psi\left(\Phi\left(\mathcal{F}_{\mathcal{K}}^{c}\right)\right)$. It is easy from the frame condition that $S \subseteq S^{\prime}$. Further, if $\exists x\left(x \leq w\right.$ and $\left.x \leq w^{\prime}\right)$, then $x S w^{\prime}$ by reflexivity, symmetry and upward closure of $S$. Thus again by upward closure of $S$, $w S w^{\prime} ;$ so $S \supseteq S^{\prime}$.

This allows us to conclude the following completeness of $\mathbf{d a C}$ with respect to the frames of $\mathbf{C C}_{\omega}$ : let us denote the derivability in daC by $\vdash_{d}$.

Corollary 5.11. $\vdash_{d} A$ if and only if $\mathcal{F}_{\mathcal{K}}^{c} \vDash_{\mathcal{K}_{c}} A$ for all $\mathcal{F}_{\mathcal{K}}^{c}$ satisfying $\forall u, v\left(u S v \rightarrow \exists w S^{-1} v(w \leq u\right.$ and $\left.w \leq v)\right)$.

Proof: The last proposition established a bijection of frames agreeing in forcing. Thus the statement follows from the completeness of daC with respect to its models [11].

We now look at the frame condition for $\sim(\sim(A \vee \sim B) \vee A) \rightarrow \sim B$.

Proposition 5.12. Let $\mathcal{F}$ be a $\mathbf{C C}_{\omega}$-frame. Then the following conditions are equivalent.

(i) $\mathcal{F} \vDash_{\mathcal{K}_{c}} \sim(\sim(A \vee \sim B) \vee A) \rightarrow \sim B$ for all $A, B$. 
(ii) $\mathcal{F}$ satisfies $\forall u, v\left(u S v \rightarrow \exists w S^{-1} v(w \leq v\right.$ and $\left.\forall x(w S x \rightarrow u S x))\right)$.

Proof: We shall first see (i) implies (ii). We show this by contraposition. Assume $u S v$ but $\neg \exists w S^{-1} v(w \leq v$ and $\forall x(w S x \rightarrow u S x))$. Choose $\mathcal{V}$ such that $\mathcal{V}(p)=\{w: w \not \leq v\}$ and $\mathcal{V}(q)=\{w: u S w\}$. Again the former set is upward closed, and the latter set is upward closed because of symmetry and upward closure of $S$. Now since $\forall w S^{-1} v(w \not \leq v$ or $\neg \forall x(w S x \rightarrow u S x))$, if the former disjunct holds then $w \in \mathcal{V}(p)$. And if the latter disjunct holds, then $\exists x(w S x$ and $\neg u S x)$. So if $x \Vdash_{\mathcal{K}_{c}} q$, then $u S x$, a contradiction. Thus $x \nVdash_{\mathcal{K}_{c}} q$ and consequently, $w \Vdash_{\mathcal{K}_{c}} \sim q$. Thus $\forall w S^{-1} v\left(w \Vdash_{\mathcal{K}_{c}} p\right.$ or $w \Vdash_{\mathcal{K}_{c}}$ $\sim q)$. Also if $v \Vdash_{\mathcal{K}_{c}} p$, then $v \not \leq v$, a contradiction. So $v \nVdash_{\mathcal{K}_{c}} p$; hence $u \Vdash_{\mathcal{K}_{c}} \sim(\sim(p \vee \sim q) \vee p)$. But if $u \Vdash_{\mathcal{K}_{c}} \sim q$, then $\exists x S^{-1} u\left(x \nVdash_{\mathcal{K}_{c}} q\right)$. So $\neg u S x$, a contradiction. Hence $u \nVdash_{\mathcal{K}_{c}} \sim q$. Thus $u \nVdash_{\mathcal{K}_{c}} \sim(\sim(p \vee \sim q) \vee p) \rightarrow \sim q$. Therefore $\not \nvdash_{\mathcal{K}_{c}} \sim(\sim(p \vee \sim q) \vee p) \rightarrow \sim q$.

To see (ii) implies (i), let $v \geq u$ for arbitrary and assume $v \Vdash_{\mathcal{K}_{c}} \sim(\sim(A \vee$ $\sim B) \vee A$ ). We want to show $v \Vdash_{\mathcal{K}_{c}} \sim B$. By definition, $\exists w S^{-1} v\left(w \nVdash_{\mathcal{K}_{c}}\right.$ $\sim(A \vee \sim B) \vee A)$. So $\forall x S^{-1} w\left(x \Vdash_{\mathcal{K}_{c}} A \vee \sim B\right)(*)$ and $w \nVdash_{\mathcal{K}_{c}} A$. By the frame condition, there is $x S^{-1} w$ such that $x \leq w$ and $\forall y(x S y \rightarrow v S y)$. From $\left(^{*}\right)$ we infer $x \Vdash_{\mathcal{K}_{c}} A$ or $x \Vdash_{\mathcal{K}_{c}} \sim B$. If the former, then $w \Vdash_{\mathcal{K}_{c}} A$, a contradiction. So $x \Vdash_{\mathcal{K}_{c}} \sim B$. But then for some $y S^{-1} x, y \nVdash_{\mathcal{K}_{c}} B$. Thus $v S y$ by the frame condition. So $v \Vdash_{\mathcal{K}_{c}} \sim B$. Hence $u \Vdash_{\mathcal{K}_{c}} \sim(\sim(A \vee \sim B) \vee$ $A) \rightarrow \sim B$. Since $u$ is arbitrary, $\vDash_{\mathcal{K}_{c}} \sim(\sim(A \vee \sim B) \vee A) \rightarrow \sim B$.

Note that contrary to the last case, in this proof we appealed to the symmetry of $S$ in $\mathbf{C C}_{\omega}$.

\section{Conclusion}

We have looked at a family of logics related to IPC ${ }^{\sim}$. In the fourth section we observed how Kripke and Beth semantics respectively reflected the (empirical) negations of $\mathbf{I P C}{ }^{\sim}$ and $\mathbf{T} \mathbf{C C}_{\omega}$, and a translation of classical logic into the latter which highlights the difference. In the fifth section, we clarified how we can eliminate the rule $[\mathrm{RP}]$ in $\mathbf{I P C} \mathbf{C}^{\sim}$ and $\mathbf{d a C}$, and how we can capture the latter logic in the setting of $\mathbf{C C}_{\omega}$. This result is further developed in the sequel, where we formulate labelled sequent calculi for the systems treated in this paper. 
Acknowledgements This research was supported by the Japan Society for the Promotion of Science (JSPS), Core-to-Core Program (A. Advanced Research Networks). The author is indebted to Hitoshi Omori and Giulio Fellin for their suggestion to look at empirical negation and co-negation, respectably. He also thanks Hajime Ishihara, Takako Nemoto and Keita Yokoyama for their encouragement and many valuable suggestions during the production of this paper. Lastly, he thanks the anonymous reviewer for the helpful comments and suggestions.

\section{References}

[1] L. E. J. Brouwer, Begründung der Mengenlehre unabhängig vom logischen Satz vom ausgeschlossenen Dritten. Zweiter Teil, [in:] A. Heyring (ed.), L.E.J. Brouwer Collected Works 1: Philosophy and Foundations of Mathematics, North-Holland (1975), pp. 191-221, DOI: http://dx.doi. org/10.1016/C2013-0-11893-4.

[2] J. L. Castiglioni, R. C. E. Biraben, Strict paraconsistency of truth-degree preserving intuitionistic logic with dual negation, Logic Journal of the IGPL, vol. 22(2) (2014), pp. 268-273, DOI: http://dx.doi.org/10.1093/ jigpal/jzt027.

[3] M. De, Empirical Negation, Acta Analytica, vol. 28 (2013), pp. 49-69, DOI: http://dx.doi.org/10.1007/s12136-011-0138-9.

[4] M. De, H. Omori, More on Empirical Negation, [in:] R. Goreé, B. Kooi, A. Kurucz (eds.), Advances in Modal Logic, vol. 10, College Publications (2014), pp. 114-133.

[5] K. Došen, Negation on the Light of Modal Logic, [in:] D. M. Gabbay, H. Wansing (eds.), What is Negation?, Kluwer Academic Publishing. (1999), DOI: http://dx.doi.org/10.1007/978-94-015-9309-0_4.

[6] T. M. Ferguson, Extensions of Priest-da Costa Logic, Studia Logica, vol. 102 (2013), pp. 145-174, DOI: http://dx.doi.org/10.1007/s11225-0139469-4.

[7] A. B. Gordienko, A Paraconsistent Extension of Sylvan's Logic, Algebra and Logic, vol. 46(5) (2007), pp. 289-296, DOI: http://dx.doi.org/10.1007/ s10469-007-0029-8.

[8] A. Heyting, Intuitionism: An Introduction, third revised ed., North Holland (1976). 
[9] M. Osorio, J. L. Carballido, C. Zepeda, J. A. Castellanos, Weakening and Extending $\mathbb{Z}$, Logica Universalis, vol. 9(3) (2015), pp. 383-409, DOI: http: //dx.doi.org/10.1007/s11787-015-0128-6.

[10] M. Osorio, J. A. C. Joo, Equivalence among RC-type paraconsistent logics, Logic Journal of the IGPL, vol. 25(2) (2017), pp. 239-252, DOI: http: //dx.doi.org/10.1093/jigpal/jzw065.

[11] G. Priest, Dualising Intuitionistic Negation, Principia, vol. 13(2) (2009), pp. 165-184, DOI: http://dx.doi.org/10.5007/1808-1711.2009v13n2p165.

[12] C. Rauszer, A formalization of the propositional calculus of $H$-B logic, Studia Logica, vol. 33(1) (1974), pp. 23-34, DOI: http://dx.doi.org/10.1007/ BF02120864.

[13] C. Rauszer, Applications of Kripke models to Heyting-Brouwer logic, Studia Logica, vol. 36(1) (1977), pp. 61-71, DOI: http://dx.doi.org/10.1007/ BF02121115.

[14] G. Restall, Extending intuitionistic logic with subtraction (1997), unpublished.

[15] R. Sylvan, Variations on da Costa C Systems and dual-intuitionistic logics I. Analyses of $C_{\omega}$ and $C C_{\omega}$, Studia Logica, vol. 49(1) (1990), pp. 47-65, DOI: http://dx.doi.org/10.1007/BF00401553.

[16] A. S. Troelstra, J. R. Moschovakis, A.S. Troelstra, D. van Dalen, Constructivism in Mathematics Corrections, URL: https://www.math.ucla.edu/ joan/ourTvDcorr030818, [accessed 20/Jul/2020].

[17] A. S. Troelstra, D. van Dalen, Constructivism in Mathematics: An Introduction, vol. I, Elsevier (1988).

[18] A. S. Troelstra, D. van Dalen, Constructivism in Mathematics: An Introduction, vol. II, Elsevier (1988).

[19] D. van Dalen, L.E.J. Brouwer: Topologist, Intuitionist, Philosopher, Springer (2013), DOI: http://dx.doi.org/10.1007/978-1-4471-4616-2.

\section{Satoru Niki}

Japan Advanced Institute of Science and Technology

School of Information Science

923-1292, 1-1 Asahidai, Nomi

Ishikawa, Japan

e-mail: satoruniki@jaist.ac.jp 